\title{
Multivariate Analysis of Malt Barley Genotypes for Different Malt Quality and Agronomic Traits in Ethiopia
}

\author{
Thomas Tsige \\ Holetta Agricultural Research Center, P.O. Box 31, Holetta, Ethiopia
}

\begin{abstract}
Barley is one of the widely grown cereal crop in the highlands of Ethiopia. Twenty five malt barley genotypes were evaluated using a $5 \times 5$ simple lattice design at Holetta, Bekoji, Debreberhan and Kofele locations to group tested malt barley genotypes, to characterize traits that contribute to total variability and to determine genetic variability among malt barley genotypes. The tested genotypes showed significant variation for all eleven agronomic and malt quality traits considered in this experiment. The candidate genotype (IBON-HI 118/2016) showed relatively better malt quality and agronomic performance. The first three principal components (PCs) contributes $85 \%$ total variability. Days to heading, maturity and malt quality traits (protein, extract and friability), plant height and grain yield contribute chiefly for $50 \%$ percent variability explained by PC 1 . Based on cluster analysis the tested genotypes grouped into three clusters (C) consisted of 15 (C-I), 8 (C-II) and 2 (C-III) genotypes. $\mathrm{C}$-I contain genotypes which had relatively better grain yield. Whereas, C-II consists of barley genotypes with better malt qualities. Thus, crossing among genotypes from these two clusters could give better genetic recombination for important malt quality and agronomic traits.
\end{abstract}

Keywords: Cluster analysis, genetic variability, principal component

DOI: $10.7176 /$ ALST/85-03

Publication date: January $31^{\text {st }} 2021$

\section{Introduction}

Barley is the fifth most important cereals of Ethiopia. It accounts for about $6.42 \%$ of the total area and $5.63 \%$ of the gross grain production of cereal crops, more than 3.7 million small holder farmers engaged in barley production (CSA, 2019). Barley has deep roots in the conception habits of Ethiopians. It used to prepare traditional foods, such as injera, besso, chiko, genfo, kolo, kinche and kitta; local beverages, tella, borde, areki and atmit. Currently, in Ethiopia, there is a high demand of malt barley with the introduction of new breweries and malt factories (Bekele et al., 2005; Kifle, 2016). This make barley an important cash crop for farmers found in high lands of the country. Malt barley genotypes have specific qualities (Low protein content (9.5-11), high extract ( $>80 \%)$, high friability $(>80 \%)$ ) required to use for malting purpose. Therefore, beside the major agronomic traits, the malt barley breeding activities focus on these quality parameters. In Ethiopia, the national research system released many malt barley varieties, where some of them are widely cultivated across the country. The Holetta Agriculture research center is one of the major breeding center for malt barley improvement. It conducts selection activities from lines derived from its own crossing activities and introduced materials from international sources. The program handles many genotypes and deal with main traits. A multivariate analysis used to evaluate such data set which contains more than two variables at once (Kumar et al., 2013). Cluster and principal component analysis are popular multivariate analysis techniques used to group and characterize genotypes. The former used to partition a set of data into clusters, where objects within the cluster are similar to one another, while dissimilar to objects in other clusters (Han et al., 2012). Principal component analysis used to reduce the dimensionality of large data sets into a smaller one that still contains most of the information in the large set (Jaadi, 2019). This study was, therefore, aimed to group malt barley genotypes into similar groups, to characterize traits that contribute to total variation and to determine genetic variability among malt barley genotypes.

\section{Materials and methods}

In this experiment, twenty five malt barley genotypes were grown in simple lattice design. The genotypes used in the present study were extracted from malt barley yield trials (national variety, preliminary variety and observation nursery trials), parental performance trial and malt barley released varieties (Table 1). Except EH 1847, Fatima and Henrike all the genotypes included in the trial serve as a parent in the 2019 off-season crossing activity. This experiment was carried out at Holetta $\left(9^{\circ} 00^{\prime} \mathrm{N}, 38^{\circ} 38^{\prime} \mathrm{E}\right)$, Bekoji $\left(7^{\circ} 15^{\prime} \mathrm{N}, 39^{\circ} 15^{\prime} \mathrm{E}\right)$, Debreberhan $\left(9^{\circ} 41^{\prime} \mathrm{N}, 39^{\circ} 32^{\prime} \mathrm{E}\right)$ and Kofele $\left(7^{\circ} 00^{\prime} \mathrm{N}, 38^{\circ} 45^{\prime} \mathrm{E}\right)$ sites during 2019 main cropping seasons. The genotypes were sown in ten-row plots each having $2.5 \mathrm{~m}$ length and $2 \mathrm{~m}$ width. In the experiment, eleven agronomic and malt quality traits were measured. These include days to $50 \%$ heading, days to $50 \%$ maturity, plant height $(\mathrm{cm})$, scald severity $(\%)$, net blotch severity $(\%)$, thousand kernel weight $(\mathrm{gm})$, hectoliter weight $\left(\mathrm{Kghl}^{-1}\right)$, grain yield $\left(\mathrm{Kgha}^{-1}\right)$, protein content $(\%)$, extract (\%), and friability (\%). Scald and net blotch disease severity recorded by visually estimating the percentage of leaf area diseased and rated using the Saari and Prescott (1975) scale. The malt quality traits were analyzed following Near infrared spectroscopy (NIRs) technique using Bruker Tango instrument at Holetta quality 
laboratory. All crop management practices were followed as per the recommendation for each location.

The analysis of variance was analyzed using lmer4 package of R-software (Douglas et al., 2015), where location and genotype considered as random and fixed effects, respectively. The mean separation was done using emmeans package of R-software (Russell, 2019). The multivariate analyses were made based on the mean values for the eleven traits and 25 barley genotypes over the four locations. For cluster and principal component analysis, the mean data were first pre-standardized to mean zero and variance unity to avoid bias due to differences in measurement scales. Multivariate statistical analysis was done using MINTAB statistical computer package, version 19 (MINITAB, 2020) and the points where local peaks of the pseudo F statistic join with small values of the pseudo $\mathrm{t}^{2}$ statistic followed by a larger pseudo $\mathrm{t}^{2}$ for the next cluster fusion were observed to decide the number of clusters (SAS Institute, 2002).

Table 1. The list of genotypes used in the study

\begin{tabular}{lll|lll}
\hline Entry & Genotype & Source & Entry & Genotype & Source \\
\hline 1 & IBON 174/03 x Traveller & MBNVT & 14 & IBON-HI 13/14 P\# 128 & NPPT \\
2 & IBON-HI13/14-49 & MBNVT & 15 & HB 1963 & Released \\
3 & IBON-HI14/15-126 & MBNVT & 16 & MN Brite & Released \\
4 & Bekoji-1 x ND 26333 & MBPVT & 17 & Traveller & Released \\
5 & Bekoji-1 x ND 24263 & MBPVT & 18 & IBON 174/03 & Released \\
6 & IBYT-HI 4/2016 & MBPVT & 19 & Miscal-21 & Released \\
7 & Bekoji-1 x Lab No. 128 & MBPVT & 20 & Acc. \#212959A & Accession line \\
8 & IBON-HI 118/2016 & MBPVT & 21 & Acc. \#212959B & Accession line \\
9 & Bekoji-1 x Traveler & MBPVT & 22 & IBON 15/2018 & MBON \\
10 & IBON 174/03 x Lab No. 128 & MBPVT & 23 & EH 1847 & Released \\
11 & Planet & Released & 24 & Fatima & Released \\
12 & G 13-64 Belgium & NPPT & 25 & Henrike & Released \\
13 & ICARDA GP-67 & NPPT & & & \\
\hline
\end{tabular}

MBNVT=Malt barley national variety trial, MBPVT=Malt barley national trial, NPPT=National parental performance trial, $\mathrm{MBON}=$ Malt barley observation nursery

\section{Results and discussion}

The analysis of variance combined over locations showed significant differences among the genotype for all traits (Table 2). This variation offers ample chances to select better genotypes for direct release or donor parent in crossing program. Similarly, mean squares due to location and genotype by location interaction were also highly significant $(\mathrm{p}<0.01)$ for all traits (Table 2). This is in line with the findings reported from evaluations of various barley genotypes for different traits (Rodriguez et al., 2008; Zerihun, 2011; Abtew et al., 2015; Thomas et al., 2019,2020; Thomas 2020).

Table 2. Mean squares from the combined analysis variance of 12 traits for 25 malt barley genotypes over four locations

\begin{tabular}{|c|c|c|c|c|c|c|c|c|c|c|c|c|}
\hline & $\overline{D f}$ & DHE & DMA & $\overline{\text { PLH }}$ & $\overline{\mathrm{SC}}$ & NB & TKW & HLW & GYLD & PR & $\overline{E X}$ & $\overline{F R}$ \\
\hline gen & 24 & $493.3^{* *}$ & $344.7^{* *}$ & $1925.4^{* *}$ & $940.9^{* *}$ & $497.9^{* *}$ & $251.84^{* *}$ & $79.4^{* *}$ & $3741306^{* *}$ & $4.431^{* *}$ & $24.4^{* *}$ & $799.39^{* *}$ \\
\hline loc & 3 & $669.8^{* *}$ & $4201.2^{* *}$ & $3176.4^{* *}$ & $11288.6^{* *}$ & $15818^{* *}$ & $1313.81^{* *}$ & $1043.4^{* *}$ & $53269595^{* *}$ & $43.91^{* *}$ & $257.1^{* *}$ & $2100.31^{* *}$ \\
\hline gen:loc & 72 & $32.5^{* *}$ & $34.6^{* *}$ & $49.3^{* *}$ & $325.3^{* *}$ & $235.8^{* *}$ & $22.26^{* *}$ & $7.6^{* *}$ & $923985^{* *}$ & $0.65^{* *}$ & $1.21^{* *}$ & $101.67^{* *}$ \\
\hline loc:rep & 4 & 7.3 & $37^{*}$ & $207.5^{* *}$ & $449.7^{*}$ & 99.3 & 5.52 & 3.88 & $911145^{*}$ & $0.96^{* *}$ & $1.59^{*}$ & 58.46 \\
\hline loc:rep:row & 32 & $9.3^{*}$ & 11.7 & $40.1^{*}$ & 247.7 & 47.5 & 10.1 & 3.7 & $516255^{*}$ & $0.70^{* *}$ & $1.02^{*}$ & 32.36 \\
\hline residuals & 64 & 5.0 & 12.6 & 21.6 & 156.4 & 58.4 & 9.46 & 3.02 & 273424 & 0.24 & 0.60 & 29.73 \\
\hline $\mathrm{CV}$ & & 2.71 & 2.62 & 5 & 25.1 & 24.4 & 7.3 & 2.8 & 17.2 & 4 & 0.96 & 8.27 \\
\hline Mean & & 82.45 & 135.5 & 92.7 & 49.8 & 31.3 & 42.2 & 63.1 & 3042.0 & 12.26 & 80.4 & 65.95 \\
\hline
\end{tabular}

*** significant at $\mathrm{P}=0.01$ and 0.05 level respectively, $\mathrm{Df}=$ degree of freedom, $\mathrm{DHE}=$ days to heading, $\mathrm{DMA}=\mathrm{days}$ to maturity, $\mathrm{PLH}=$ plant height, $\mathrm{SC}=$ scald severity, $\mathrm{NB}=$ net blotch, $\mathrm{TKW}=$ thousand kernel weight, $\mathrm{HLW}=$ hectoliter weight, $\mathrm{GYLD}=$ grain yield, $\mathrm{PR}=$ protein, $\mathrm{EX}=$ Extract, $\mathrm{FR}=$ Friability

Significant mean differences were observed among the barley varieties for grain yield. Entry \#10 and \#18 demonstrated top ranking grain yield performance, though not significantly different from the other 15 released and elite materials included in the trial (Table 3). Concerning malt quality traits ICARDA GP-67, HB 1963, 
Traveller, Planet, IBON-HI 118/2016 and Fatima showed good performance (Table 3). In addition, there was a large variability in mean plant height, which ranged from 62-115 cm. Mostly, the imported malt barley genotypes had relatively less resistance to scaled and the two accession lines (Entry \#20 and \#21) showed less resistance to net blotch. On the other hand, IBON-HI 13/14 P\# 128, Fatima, Traveller, Planet and ICARDA GP-67 had the maximum heading date, but the released variety IBON 174/03 and its crosses had early heading date. Regarding malt quality traits Planet, Fatima, Traveller and HB 1963 showed good performance (Table 3). So, these genotypes can serve as parents in the malt barley crossing program. Moreover, the candidate genotypes IBON-HI 118/2016, which had good malt quality and agronomic performance can be recommended for variety verification trial.

Table 3. Overall means for eleven traits of 25 malt barley genotypes tested during 2019 main season at Holetta, Bekoji, Debreberhan and Kofele locations

\begin{tabular}{|c|c|c|c|c|c|c|c|c|c|c|c|c|}
\hline Entry & Genotype & DHE & DMA & PLH & $\mathrm{SC}$ & NB & TKW & HLW & GYLD & $\mathbf{P R}$ & $\mathbf{E X}$ & $\overline{\text { FR }}$ \\
\hline 1 & IBON 174/03 x Traveller & $71^{\mathrm{jk}}$ & $130^{\mathrm{def}}$ & $99^{\mathrm{c}-\mathrm{f}}$ & $44.8^{\mathrm{d}-\mathrm{h}}$ & $27.5^{\text {cde }}$ & $46.6^{\mathrm{abc}}$ & $62.6^{\mathrm{f}-\mathrm{j}}$ & $3806^{\mathrm{abc}}$ & $12.6^{\mathrm{a}-\mathrm{e}}$ & $78.9^{\mathrm{k}-\mathrm{n}}$ & $53.2^{\mathrm{kl}}$ \\
\hline 2 & IBON-HI13/14-49 & $76^{\mathrm{h}-\mathrm{k}}$ & $131^{\mathrm{c}-\mathrm{f}}$ & $97^{\mathrm{d}-\mathrm{g}}$ & $32.9^{\mathrm{gh}}$ & $36.2^{\mathrm{a}-\mathrm{d}}$ & $46.2^{\mathrm{a}-\mathrm{d}}$ & $63.3^{\mathrm{b}-\mathrm{j}}$ & $3215^{\mathrm{a}-\mathrm{f}}$ & $12.5^{\mathrm{b}-\mathrm{f}}$ & $80.8^{\mathrm{fgh}}$ & $60.5^{\mathrm{ijk}}$ \\
\hline 3 & IBON-HI14/15-126 & $80^{\mathrm{e}-\mathrm{h}}$ & $136^{\mathrm{bcd}}$ & $97^{\mathrm{d}-\mathrm{g}}$ & $28.4^{\mathrm{h}}$ & $32.4^{\mathrm{b}-\mathrm{e}}$ & $38.6^{\mathrm{fg}}$ & $65.9^{\mathrm{a}-\mathrm{d}}$ & $3507^{\mathrm{a}-\mathrm{f}}$ & $12.1^{\mathrm{d}-\mathrm{h}}$ & $79.2^{\mathrm{j}-\mathrm{m}}$ & $71.5^{\mathrm{c}-\mathrm{g}}$ \\
\hline 4 & Bekoji-1 x ND 26333 & $85^{\text {cde }}$ & $137^{\mathrm{b}}$ & $110^{\mathrm{ab}}$ & $38.2^{\mathrm{fgh}}$ & $36.4^{\mathrm{a}-\mathrm{d}}$ & $47.0^{\mathrm{ab}}$ & $66.3^{\mathrm{ab}}$ & $2999^{b-h}$ & $12.6^{\mathrm{a}-\mathrm{e}}$ & $80.4^{\mathrm{f}-\mathrm{i}}$ & $64.2^{e-j}$ \\
\hline 5 & Bekoji-1 x ND 24263 & $84^{\text {def }}$ & $138^{\mathrm{b}}$ & $114^{\mathrm{a}}$ & $39.6^{\text {fgh }}$ & $32.6^{\mathrm{b}-\mathrm{e}}$ & $46.0^{\mathrm{a}-\mathrm{d}}$ & $65.4^{\mathrm{a}-\mathrm{g}}$ & $3091^{\mathrm{b}-\mathrm{g}}$ & $13.2^{\mathrm{ab}}$ & $80.2^{g-j}$ & $60.0^{\mathrm{ijk}}$ \\
\hline 6 & IBYT-HI 4/2016 & $77^{\text {gh }}$ & $133^{\mathrm{b}-\mathrm{e}}$ & $90^{\text {ghi }}$ & $44.4^{\mathrm{d}-\mathrm{h}}$ & $21.3^{\mathrm{de}}$ & $43.3^{\mathrm{b}-\mathrm{e}}$ & $64.3^{\mathrm{a}-\mathrm{h}}$ & $3018^{\mathrm{b}-\mathrm{h}}$ & $12.2^{\mathrm{d}-\mathrm{h}}$ & $79.5^{\mathrm{i}-1}$ & $59.8^{\mathrm{ijk}}$ \\
\hline 7 & Bekoji-1 x Lab No. 128 & $85^{\text {cde }}$ & $135^{\mathrm{bcd}}$ & $115^{\mathrm{a}}$ & $46.9^{\mathrm{c}-\mathrm{g}}$ & $32.6^{\mathrm{b}-\mathrm{e}}$ & $44.5^{\mathrm{bcd}}$ & $65.9^{\mathrm{a}-\mathrm{e}}$ & $3426^{\mathrm{a}-\mathrm{f}}$ & $12.8^{\mathrm{a}-\mathrm{d}}$ & $80.5^{\mathrm{f}-\mathrm{i}}$ & $61.0^{\mathrm{h}-\mathrm{k}}$ \\
\hline 8 & IBON-HI 118/2016 & $82^{\text {efg }}$ & $130^{\text {def }}$ & $103^{\text {bcd }}$ & $51.7^{\mathrm{b}-\mathrm{f}}$ & $33.8^{\mathrm{b}-\mathrm{e}}$ & $44.5^{\mathrm{bcd}}$ & $62.4^{\mathrm{g}-\mathrm{j}}$ & $3159^{\mathrm{a}-\mathrm{g}}$ & $11.8^{\mathrm{f}-\mathrm{j}}$ & $81.1^{\mathrm{efg}}$ & $80.7^{\mathrm{abc}}$ \\
\hline 9 & Bekoji-1 x Traveler & $81^{\mathrm{e}-\mathrm{h}}$ & $137^{\mathrm{b}}$ & $109^{\mathrm{ab}}$ & $50.8^{\mathrm{b}-\mathrm{f}}$ & $27.5^{\text {cde }}$ & $46.0^{\mathrm{a}-\mathrm{d}}$ & $66.0^{\mathrm{abc}}$ & $3254^{\mathrm{a}-\mathrm{f}}$ & $12.1^{\mathrm{d}-\mathrm{h}}$ & $81.3^{\mathrm{def}}$ & $61.6^{\mathrm{g}-\mathrm{k}}$ \\
\hline 10 & IBON 174/03 x Lab No. 128 & $71^{\mathrm{k}}$ & $133^{\mathrm{b}-\mathrm{e}}$ & $94^{\mathrm{e}-\mathrm{h}}$ & $61.1^{\mathrm{a}-\mathrm{d}}$ & $27.5^{\text {cde }}$ & $46.6^{\mathrm{abc}}$ & $63.0^{\mathrm{c}-\mathrm{j}}$ & $4073^{\mathrm{a}}$ & $12.6^{\mathrm{a}-\mathrm{e}}$ & $78.4^{\mathrm{mno}}$ & $56.1^{\mathrm{jk} 1}$ \\
\hline 11 & Planet & $93^{\mathrm{ab}}$ & $146^{\mathrm{a}}$ & $68^{\mathrm{kl}}$ & $65.0^{\mathrm{ab}}$ & $21.4^{\mathrm{de}}$ & $38.8^{\mathrm{fg}}$ & $62.7^{\mathrm{e}-\mathrm{j}}$ & $1859^{\mathrm{ijk}}$ & $10.1^{\mathrm{k}}$ & $83.4^{\mathrm{ab}}$ & $89.9^{\mathrm{a}}$ \\
\hline 12 & G 13-64 Belgium & $78^{\mathrm{gh}}$ & $133^{\mathrm{b}-\mathrm{e}}$ & $101^{\mathrm{cd}}$ & $39.6^{\mathrm{fgh}}$ & $33.9^{\mathrm{b}-\mathrm{e}}$ & $47.2^{\mathrm{ab}}$ & $66.0^{\mathrm{abc}}$ & $2924^{c-h}$ & $12.3^{\mathrm{c}-\mathrm{g}}$ & $80.0^{\text {hij }}$ & $59.6^{\mathrm{ijk}}$ \\
\hline 13 & ICARDA GP-67 & $93^{\mathrm{ab}}$ & $145^{\mathrm{a}}$ & $71^{\mathrm{jk}}$ & $44.6^{\mathrm{d}-\mathrm{h}}$ & $41.2^{\mathrm{abc}}$ & $41.7^{\mathrm{d}-\mathrm{g}}$ & $64.1^{\mathrm{a}-\mathrm{i}}$ & $2069^{h-k}$ & $12.0^{\mathrm{e}-\mathrm{i}}$ & $82.1^{\text {cde }}$ & $72.2^{\mathrm{c}-\mathrm{f}}$ \\
\hline 14 & IBON-HI 13/14 P\# 128 & $98^{\mathrm{a}}$ & $146^{\mathrm{a}}$ & $62^{1}$ & $58.4^{\mathrm{a}-\mathrm{e}}$ & $36.4^{\mathrm{a}-\mathrm{d}}$ & $33.2^{\mathrm{hi}}$ & $60.9^{\mathrm{ij}}$ & $1706^{\mathrm{k}}$ & $11.5^{\text {hij }}$ & $82.3^{\mathrm{bcd}}$ & $83.6^{\mathrm{ab}}$ \\
\hline 15 & НВ 1963 & $88^{\mathrm{bcd}}$ & $144^{\mathrm{a}}$ & $101^{\mathrm{cd}}$ & $46.5^{\mathrm{c}-\mathrm{g}}$ & $27.5^{\text {cde }}$ & $46.3^{\mathrm{abc}}$ & $67.4^{\mathrm{a}}$ & $2922^{\mathrm{c}-\mathrm{h}}$ & $11.1^{\mathrm{j}}$ & $81.9^{\text {cde }}$ & $71.0^{\mathrm{c}-\mathrm{h}}$ \\
\hline 16 & MN Brite & $79^{\mathrm{fgh}}$ & $133^{\mathrm{b}-\mathrm{e}}$ & $86^{\mathrm{i}}$ & $59.0^{\mathrm{a}-\mathrm{d}}$ & $23.7^{\mathrm{de}}$ & $38.0^{\mathrm{fg}}$ & $65.8^{\mathrm{a}-\mathrm{f}}$ & $3752^{\mathrm{a}-\mathrm{d}}$ & $12.6^{\mathrm{a}-\mathrm{e}}$ & $80.2^{\mathrm{g}-\mathrm{j}}$ & $57.4^{\mathrm{i}-1}$ \\
\hline 17 & Traveller & $93^{\mathrm{ab}}$ & $146^{\mathrm{a}}$ & $72^{\mathrm{jk}}$ & $50.6^{\mathrm{b}-\mathrm{f}}$ & $36.3^{\mathrm{a}-\mathrm{d}}$ & $45.1^{\mathrm{a}-\mathrm{d}}$ & $62.7^{\mathrm{d}-\mathrm{j}}$ & $2795^{\mathrm{d}-\mathrm{i}}$ & $11.7^{g-j}$ & $82.5^{\mathrm{abc}}$ & $74.3^{\mathrm{b}-\mathrm{e}}$ \\
\hline 18 & IBON 174/03 & $72^{\mathrm{ijk}}$ & $130^{\mathrm{def}}$ & $93^{\mathrm{fgh}}$ & $48.4^{\mathrm{b}-\mathrm{g}}$ & $28.6^{\text {cde }}$ & $44.3^{\mathrm{bcd}}$ & $62.3^{\mathrm{g}-\mathrm{j}}$ & $3961^{\mathrm{ab}}$ & $13.0^{\mathrm{ab}}$ & $77.9^{\text {no }}$ & $49.0^{1}$ \\
\hline 19 & Miscal-21 & $77^{\mathrm{g}-\mathrm{j}}$ & $130^{\text {def }}$ & $103^{\text {bcd }}$ & $51.6^{\mathrm{b}-\mathrm{f}}$ & $31.3^{\text {cde }}$ & $45.1^{\mathrm{a}-\mathrm{d}}$ & $63.5^{\mathrm{b}-\mathrm{i}}$ & $3670^{\mathrm{a}-\mathrm{e}}$ & $13.3^{\mathrm{a}}$ & $79.8^{\mathrm{h}-\mathrm{k}}$ & $57.8^{i-1}$ \\
\hline 20 & Acc. \#212959A & $77^{\mathrm{g}-\mathrm{j}}$ & $126^{\mathrm{f}}$ & $100^{\text {cde }}$ & $47.0^{\mathrm{c}-\mathrm{g}}$ & $50.0^{\mathrm{a}}$ & $28.0^{\mathrm{j}}$ & $51.1^{\mathrm{k}}$ & $2750^{\mathrm{e}-\mathrm{i}}$ & $13.3^{\mathrm{a}}$ & $77.5^{\circ}$ & $62.5^{\mathrm{f}-\mathrm{k}}$ \\
\hline 21 & Acc. \#212959B & $77^{\mathrm{gh}}$ & $125^{\mathrm{f}}$ & $97^{\mathrm{d}-\mathrm{g}}$ & $41.4^{\mathrm{e}-\mathrm{h}}$ & $47.5^{\mathrm{ab}}$ & $29.5^{\mathrm{ij}}$ & $53.3^{k}$ & $2597^{\mathrm{f}-\mathrm{k}}$ & $12.9^{\mathrm{abc}}$ & $77.9^{\text {no }}$ & $64.2^{\mathrm{e}-\mathrm{j}}$ \\
\hline 22 & IBON 15/2018 & $85^{\text {cde }}$ & $137^{\mathrm{b}}$ & $87^{\mathrm{hi}}$ & $65.5^{\mathrm{ab}}$ & $20.1^{\mathrm{e}}$ & $49.0^{\mathrm{a}}$ & $62.8^{\mathrm{c}-\mathrm{j}}$ & $1771^{\mathrm{jk}}$ & $11.8^{\mathrm{f}-\mathrm{j}}$ & $78.6^{\mathrm{lmn}}$ & $66.4^{\mathrm{d}-\mathrm{i}}$ \\
\hline 23 & EH 1847 & $77^{\mathrm{ghi}}$ & $129^{\mathrm{ef}}$ & $105^{\mathrm{bc}}$ & $55.7^{\mathrm{a}-\mathrm{f}}$ & $33.7^{\mathrm{b}-\mathrm{e}}$ & $42.3^{\mathrm{c}-\mathrm{f}}$ & $64.6^{\mathrm{a}-\mathrm{h}}$ & $3597^{\mathrm{a}-\mathrm{e}}$ & $13.2^{\mathrm{a}}$ & $80.3^{\mathrm{f}-\mathrm{i}}$ & $60.8^{\mathrm{ijk}}$ \\
\hline 24 & Fatima & $96^{\mathrm{a}}$ & $144^{\mathrm{a}}$ & $66^{\mathrm{kl}}$ & $63.3^{\mathrm{abc}}$ & $23.9^{\text {de }}$ & $38.8^{\text {efg }}$ & $62.0^{\text {hij }}$ & $2191^{\mathrm{g}-\mathrm{k}}$ & $11.2^{\mathrm{ij}}$ & $83.5^{\mathrm{a}}$ & $76.5^{\mathrm{bcd}}$ \\
\hline 25 & Henrike & $89^{\mathrm{bc}}$ & $136^{\mathrm{bc}}$ & $77^{\mathrm{j}}$ & $70.2^{\mathrm{a}}$ & $18.8^{\mathrm{e}}$ & $37.3^{\text {gh }}$ & $60.1^{\mathrm{j}}$ & $2742^{e-j}$ & $11.9^{\mathrm{e}-\mathrm{i}}$ & $82.4^{\mathrm{bcd}}$ & $74.8^{\text {bcd }}$ \\
\hline & $\mathrm{CV}$ & 2.7 & 2.6 & 5.0 & 25.1 & 24.4 & 7.3 & 2.8 & 17.2 & 4.0 & 0.96 & 8.27 \\
\hline & Mean & 82.5 & 135.5 & 92.7 & 49.8 & 31.3 & 42.2 & 63.1 & 3042 & 12.26 & 80.4 & 65.95 \\
\hline
\end{tabular}

$\mathrm{DHE}=$ days to heading, $\mathrm{DMA}=$ days to maturity, $\mathrm{PLH}=$ plant height, $\mathrm{SC}=$ scald severity, $\mathrm{NB}=$ net blotch, $\mathrm{TKW}=$ thousand kernel weight, HLW= hectoliter weight, $\mathrm{GYLD}=$ grain yield, $\mathrm{PR}=$ protein, $\mathrm{EX}=$ Extract, $\mathrm{FR}=$ Friability

The cluster analysis based on average linkage method and Euclidean distance measure group 25 tested genotypes to three major clusters (Figure 1). The largest cluster (C-1) contains 15 genotypes which include all genotypes selected from preliminary and national variety yield trials. In addition, this cluster contains nationally released malt barley varieties, IBON 174/03, Miscal-21, EH 1847 and MN Brite. The second cluster comprised of the registered malt barley genotypes Planet, Fatima, Henrike and Traveller (Table 4). Recently released popular malt barley genotype (HB 1963) and other three genotypes selected from ICARDA materials also included in C2. The third cluster (C-3) contains two accession lines selected from barley acid tolerance screening trial (Table 4). Similarly, Amabile et al., 2014, group thirteen malt barley genotypes in two similarity group. However, Enyew et al. (2019), Gupta et al. (2009) and Mekonnon et al. (2014), group 48, 207 and 102 barley landrace accessions in six and five distinct clusters, respectively. Generally, the cluster analysis group the genotypes of similar origin in the same cluster and these genotypes assembled in one cluster had similar agronomic performance and malt quality. In addition, the lower number of clusters reported in the present study were could be due to selections 
applied on previous generations of these tested genotypes using common traits.

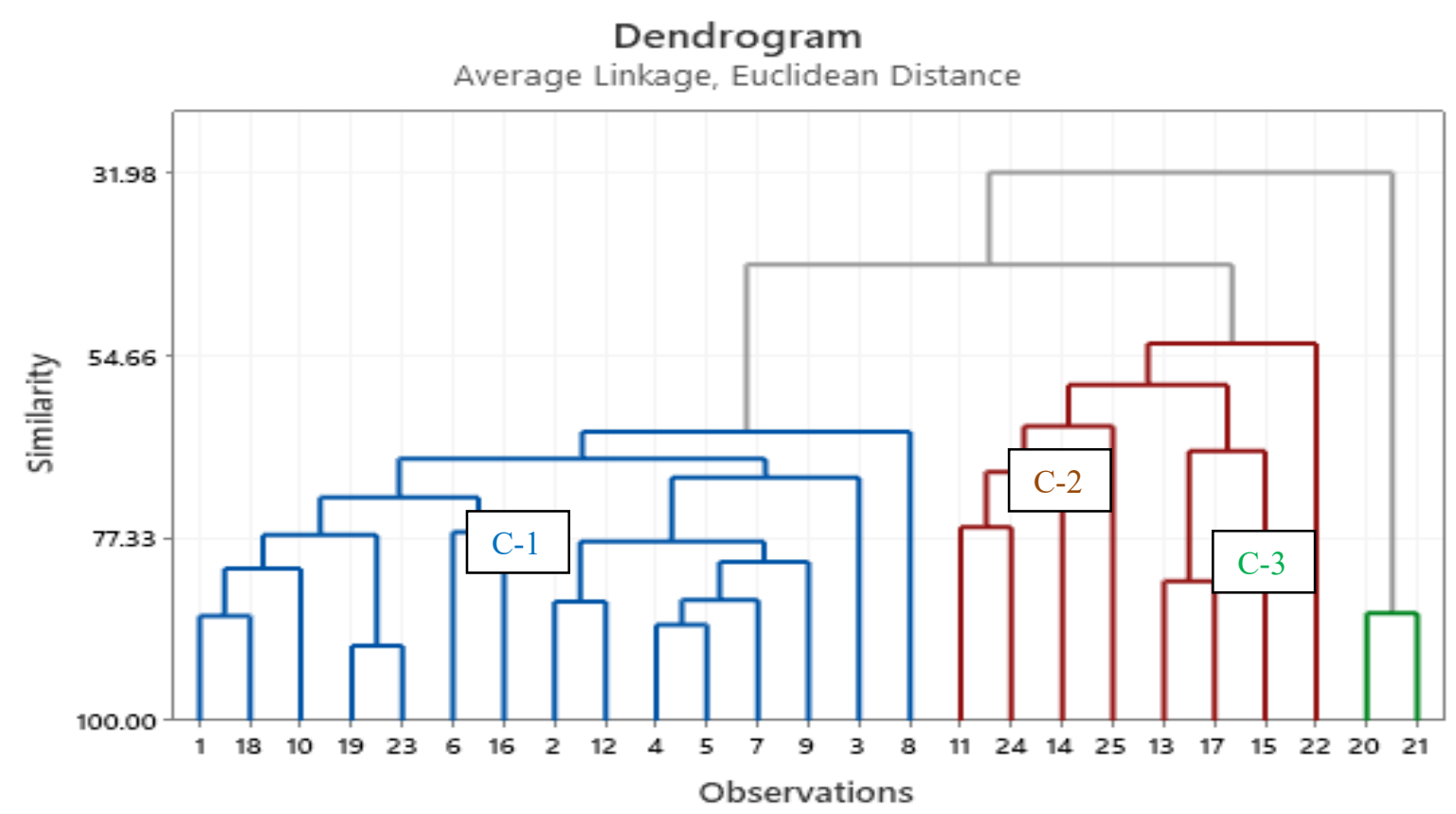

Figure 1. Dendrogram of seventy genotypes based on average linkage and Euclidean distance of 11 traits evaluated at four locations

Table 4. List of barley genotypes grouped in three clusters (average linkage euclidean distance cluster analyses) using 11 traits evaluated at four locations

\begin{tabular}{lll}
\hline Clusters & $\begin{array}{l}\text { No. of } \\
\text { genotypes }\end{array}$ & Name of tested malt barley genotypes \\
\hline I & 15 & IBON 174/03 x Traveller, IBON 174/03, IBON 174/03 x Lab No. 128, Miscal-21, \\
& & $\begin{array}{l}\text { EH 1847, IBYT-HI 4/2016, MN Brite, IBON-HI13/14-49, G 13-64 Belgium, } \\
\text { Bekoji-1 x ND 26333, Bekoji-1 x ND 24263, Bekoji-1 x Lab No. 128, Bekoji-1 x } \\
\text { Traveler, IBON-HI14/15-126 and IBON-HI 118/2016 }\end{array}$ \\
& & $\begin{array}{l}\text { Planet, Fatima, IBON-HI 13/14 P\# 128, Henrike, ICARDA GP-67, Traveller, HB } \\
\text { II }\end{array}$ \\
& 8 & Acc. \#212959A and Acc. \#212959B \\
III & 2 & And IBON 15/2018
\end{tabular}

Based on cluster mean analysis, cluster II consisted of barley genotypes with late spike emergence and maturity (Table 5). This cluster consists of genotypes having good malt qualities (protein, extract, friability), lower grain yield and relatively susceptible to scald. Whereas, cluster I showed the highest mean grain yield and plant height values. Cluster III, which contained of two accession lines, characterized by low malt quality and high net blotch values (Table 5). Generally, cluster mean values confirmed that these genotypes which had higher malt qualities showed a low mean grain yield and vice versa. Consequently, crosses among genotypes found in these different clusters could give genotypes which have a good agronomic performance and malt qualities in the subsequent segregating generations.

Table 5. Mean values of three cluster for the 11 quantitative traits

\begin{tabular}{llllllllllll}
\hline Entry & DHE & DMA & PLH & SC & NB & TKW & HLW & GYLD & PR & EX & FR \\
\hline Cluster I & 78 & 133 & 101 & 46.2 & 30.6 & 44.4 & 64.5 & 3430 & 12.6 & 79.9 & 60.9 \\
Cluster II & 92 & 143 & 76 & 58.0 & 28.2 & 41.3 & 62.8 & 2257 & 11.4 & 82.1 & 76.1 \\
Cluster III & 77 & 125 & 98 & 44.2 & 48.7 & 28.7 & 52.2 & 2674 & 13.1 & 77.7 & 63.4 \\
\hline
\end{tabular}

$\mathrm{DHE}=$ days to heading, $\mathrm{DMA}=$ days to maturity, $\mathrm{PLH}=$ plant height, $\mathrm{SC}=$ scald severity, $\mathrm{NB}=$ net blotch, $\mathrm{TKW}=$ thousand kernel weight, $\mathrm{HLW}=$ hectoliter weight, $\mathrm{GYLD}=$ grain yield, $\mathrm{PR}=$ protein, $\mathrm{EX}=$ Extract, $\mathrm{FR}=\mathrm{Friability}$

In principal component analysis (PCA), $85 \%$ of the total variation were explained by the first three principal components (PCs) and these PCs have Eigenvalue greater than one (Table 6). PC1 accounted for $50 \%$ of the variation among the genotypes under investigation. Days to heading, maturity and malt quality traits (protein, extract and friability), plant height and grain yield contribute more for the percent variability explained by PC 1 . Besides, PC2 contributed about $23 \%$ of the total variation originated mainly from variation in hectoliter weight, 
thousand kernel weight and net blotch severity. The third PC explained $12 \%$ of the variations observed among 25 malt barley genotypes. Scald and net blotch severity contribute largely to these variability (Table 5). Likewise, in Enyew et al. (2019) study the first PC alone explained about $50 \%$ of the total variance mainly due heading date, plant height and grain yield, biomass yield. However, Abebe et al., (2010) and Enyew et al. (2019) reported more contribution of thousand kernel weight for percent variation explained by PC1.

Table 6. Eigenvectors and eigenvalues of the first three principal components for 11 traits of 25 malt barley genotypes evaluated at four locations

\begin{tabular}{llll}
\hline Variable & PC1 & PC2 & PC3 \\
\hline DHE & $\mathbf{0 . 3 9 4}$ & -0.043 & -0.210 \\
DMA & $\mathbf{0 . 3 7 1}$ & 0.162 & -0.217 \\
PLH & $\mathbf{- 0 . 3 3 6}$ & 0.159 & -0.235 \\
SC & 0.220 & -0.003 & 0.683 \\
NB & -0.135 & -0.421 & -0.528 \\
TKW & -0.066 & 0.559 & -0.096 \\
HLW & 0.036 & 0.584 & -0.214 \\
GYLD & $\mathbf{- 0 . 3 3 5}$ & 0.233 & 0.122 \\
PRO & $\mathbf{- 0 . 3 8 0}$ & -0.096 & -0.015 \\
EXT & $\mathbf{0 . 3 5 8}$ & 0.180 & -0.144 \\
FRI & $\mathbf{0 . 3 7 1}$ & -0.142 & -0.132 \\
\hline Eigen value & 5.48 & 2.51 & 1.30 \\
Proportion & 0.50 & 0.23 & 0.12 \\
Cumulative & 0.50 & 0.73 & 0.85 \\
\hline
\end{tabular}

\section{Conclusion}

The tested genotypes showed significant variation for all traits, which helps to select genotypes for direct release or donor parents in crossing program. The significant genotype by environment interactions of all traits indicated that the performance of genotypes was not consistent across test locations. Besides, the principal components analysis showed that malt quality traits (protein, extract, friability), phenological traits, plant height and grain yield chiefly contribute for variation recorded by PC 1. The cluster analysis grouped twenty five barley genotypes in three clusters. Genotypes found in same cluster have similar performance, cluster I contain relatively high yielding genotypes, whereas high malt quality yielding genotypes found in cluster II. The crosses between these genotypes found in different clusters could result better segregates which had good malt and agronomic performance.

\section{References}

Abebe, T.D., Bauer, A.M. and Léon, J., 2010. Morphological diversity of Ethiopian barleys (Hordeum vulgare L.) in relation to geographic regions and altitudes. Hereditas, 147(4), pp.154-164.

Abtew, W.G., Lakew, B., Haussmann, B.I. and Schmid, K.J., 2015. Ethiopian barley landraces show higher yield stability and comparable yield to improved varieties in multi-environment field trials. Journal of plant breeding and crop science, 7(8), pp.275-291.

Amabile, R.F., Faleiro, F.G., Capettini, F., Sayd, R.M., Peixoto, J.R. and Guercia, R.F., 2014. Characterization and genetic variability of barley accessions (Hordeum vulgare L.) irrigated in the savannas based on malting quality traits. Journal of the Institute of Brewing, 120(4), pp.404-414.

Bekele, B., Alemayehu, F. and Lakew, B., 2005. Food barley in Ethiopia. Food Barley: Importance, Uses, and Local Knowledge. In: Grando, Stefania and Helena Gormez Macpherson (eds.). Food Barley: Importance, Uses and Local Knowledge. Proceedings of the International Workshop on Food Barley Improvement, 14-17 January 2002, Hammamet, Tunisia. ICARDA, Aleppo, Syria, pp.53-82.

Douglas Bates, Martin Maechler, Ben Bolker, Steve Walker (2015). Fitting Linear Mixed-Effects Models Using lme4.Journal of Statistical Software, 67(1), 1-48.doi:10.18637/jss.v067.i01.

Enyew, M., Dejene, T., Lakew, B. and Worede, F., 2019. Clustering and principal component analysis of Barley (Hordeum volugare L.) Landraces for major morphological traits from North Western Ethiopia. International Journal of Agricultural Science and Food Technology, 5(1), pp.058-063.

Gupta, S.R., Upadhyay, M.P. and Shah, U.S., 2009. Agro-morphological variability study of barley (Hordeum vulgare L.) landraces in Jumla, Nepal. Nepal Agriculture Research Journal, 9, pp.1-11.

Han, J., Kamber, M. and Pei, J., 2012. 10-cluster analysis: Basic concepts and methods. In Data mining (pp. 443495). Morgan Kaufmann.

Jaadi, Z., 2019. A step by step explanation of Principal Component Analysis. Towards Data Science. Kifle, S.M., 2016. Review on Barley Production and Marketing in Ethiopia. Journal of Economics and Sustainable Development, 7(9), pp.91-100.

Kifle, S.M., 2016. Review on Barley Production and Marketing in Ethiopia. Journal of Economics and Sustainable 
Development, 7(9), pp.91-100.

Kumar, S., Singh, S.K. and Mishra, P., 2013. Multivariate Analysis: An Overview. Journal of Dentofacial Science, 2(3), pp.19-26.

Mekonnon, B., Lakew, B. and Dessalegn, T., 2015. Morphological diversity and association of traits in Ethiopian food barley (Hordeum vulgare L.) landraces in relation to regions of origin and altitudes. Journal of Plant Breeding and Crop Science, 7(2), pp.44-54.

Minitab, 2020. Minitab ${ }^{\circledR}$ Statistical Software, Version 19. URL: www.minitab.com

Rodriguez, M., Rau, D., Papa, R. and Attene, G., 2008. Genotype by environment interactions in barley (Hordeum vulgare L.): different responses of landraces, recombinant inbred lines and varieties to Mediterranean environment. Euphytica, 163(2), pp.231-247.

Russell Lenth (2019). emmeans: Estimated Marginal Means, aka Least-Squares Means. R package version 1.3.5.1.https://CRAN.R-project.org/package=emmeans

Saari, E.E. and J.M. Prescott. 1975. A scale for appraising the foliar intensity of wheat diseases. Plant Dis. Rep. 59(5): 377-380.

SAS Institute. 2002. Proprietary Software version 9.00, Cary, NC, USA

Thomas Tsige, Tigist Shiferaw, Wondimu Fekadu, Berhane Lakew, Shimles Gezahegn, Kefyalew Taye, Workineh Mekasa, Anberber Haile and Seid Ahmed, 2019. Registration of Two Food Barley (Hordeum vulgare L.) Varieties (HB 1965 and HB1966) for the Highlands of Ethiopia. Ethiopian Journal of Applied Science and Technology, 10(1), pp.16-27.

Thomas Tsige, Tigist Shiferaw, Shimles Gezahegn, Kefyalew Taye, 2020.Assessment of Malt Barley Genotypes for Grain Yield and Malting Quality Traits in the Central Highlands of Ethiopia. Journal of Biology, Agriculture and Healthcare, 10(20), pp. 1-7.

Thomas Tsige, 2020. Genotype by environment interaction and association among traits of barley genotypes evaluated in central and south eastern Ethiopia. International Journal of Novel Research in Life Sciences, 7(4), pp. 5-30.

Zerihun, J., 2011. GGE-biplot analysis of multi-environment yield trials of barley (Hordeium vulgare L.) genotypes in Southeastern Ethiopia highlands. International journal of plant breeding and genetics, 5(1), pp.59-75. 